\title{
Diagnosis of amniotic fluid embolism using an antiserum to human keratin
}

\author{
IWC GARLAND, WD THOMPSON \\ From the Department of Pathology, University Medical Buildings, Foresterhill, Aberdeen AB9 2ZD
}

SUMMARY Sections of lung from four maternal deaths have been stained with an antibody raised to human keratin. This method has been found to be a more sensitive technique for determining amniotic fluid emboli than other special stains and has shown a higher incidence than expected.

Maternal deaths are uncommon and of these, amniotic fluid embolism is said to account for $6.4 \%$. $^{1}$ The incidence, in England and Wales, of amniotic fluid embolism is 1 per 80000 deliveries. ${ }^{2}$ Histological confirmation of this diagnosis requires adequate sampling of the lungs and is said to be made easier by the use of special staining techniques for amniotic fluid-for example, Attwood's stain. ${ }^{3}$ However, despite the use of such special stains, identification of fetal tissue in lung sections may be difficult. ${ }^{4}$

As a more sensitive method of demonstrating amniotic squames within the pulmonary circulation, the immunoperoxidase technique was employed to stain sections of lung tissue from four consecutive maternal deaths, using an antiserum raised to human keratin proteins.

\section{Case reports and methods}

CASE 1

A 27 -year-old para $1^{+1}$ was admitted, at term +12 , for induction of labour. She had had an uneventful pregnancy. After artificial rupture of the membranes she had a cardiac arrest. She was delivered of a stillborn baby after internal podalic version. She began to bleed heavily and there was laboratory evidence of disseminated intravascular coagulation (DIC). Treatment with fresh frozen plasma and whole blood improved the coagulation profile but the arterial blood pressure was not maintained. Resuscitative measures were discontinued $8 \mathrm{~h}$ after the initial cardiac arrest. The clinical diagnosis was of amniotic fluid embolism.

Post-mortem histological examination showed fibrin thrombi in renal capillaries, confirming DIC. Amniotic fluid embolism was also confirmed histologically.

Accepted for publication 16 December 1982
CASE 2

A 31-year-old para $6^{+0}$ was admitted in the second stage of labour at $35 \mathrm{wk}$ gestation. She had had no antenatal care. Three days before admission she had had an episode of chest pain and cough with copious blood-stained sputum. She was delivered, normally, of a live baby girl who died of hyaline membrane disease two days later. Post partum she developed increasing dyspnoea and bleeding from vagina and into skin. Clinically she had DIC and bilateral pneumonia. She then developed respiratory failure, acute renal failure and finally a cardiac arrest. She died four days after delivery. The clinical diagnosis was of DIC and bronchopneumonia. The postmortem examination confirmed, on histological examination, the presence of DIC, and, in addition, interstitial viral pneumonia, a uraemic pneumonitis and a terminal monilial bronchopneumonia. Histological evidence of amniotic fluid embolism was not detected.

\section{CASE 3}

A 42-year-old para $0^{+1}$ was admitted with preeclampsia at 36 wk gestation. She was delivered of a live baby girl by caesarean section but $3 \frac{1}{2}$ hours later complained of sudden chest pain. She went on to develop DIC which was treated with blood and fresh frozen plasma. Twelve hours later she was unconscious and apnoeic; her pupils were fixed and dilated and she had a grade IV hypertensive retinopathy. She died $36 \mathrm{~h}$ after delivery. Postmortem examination showed the histological changes consistent with pre-eclamptic toxaemia. Death was due to intracerebral haemorrhage associated with pre-eclamptic toxaemia and DIC. Although amniotic debris was identified in the pulmonary circulation it was not considered to be a major contributory factor. 


\section{CASE 4}

A 35-year-old woman was admitted in established labour. Prior to admission she had a vague history of diarrhoea and vomiting. She was hypotensive and "collapsed" 45 min after delivery of a stillborn infant. A clinical diagnosis of amniotic fluid embolism was made and there was haematological evidence of DIC. Despite treatment she developed pulmonary insufficiency and renal failure and died six days after delivery.

Post-mortem confirmed the presence of DIC attributed to some infective process and there was no histological evidence of amniotic fluid embolism.

\section{PREPARATION OF ANTISERUM}

Total human keratin was extracted from foot callus, collected at a chiropody clinic by the method of Fuchs and Green. ${ }^{5}$ Stratum corneum $(600 \mathrm{mg})$ was homogenised in $20 \mathrm{mmol}$ Tris $\mathrm{HCl}, \mathrm{pH} \mathrm{7.4}$, and centrifuged for $20 \mathrm{~min}$ at $12000 \mathrm{~g}$. The pellet was

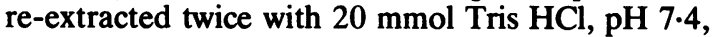
and twice with $20 \mathrm{mmol}$ Tris, $\mathrm{pH} 7.4$ containing $8 \mathrm{M}$ urea. Finally the insoluble pellet was extracted with $2 \%$ sodium dodecylsulphate (SDS) and $10 \mathrm{mmol}$ dithiothreitol, using an MSE sonicator $(4 \times 30 \mathrm{~s})$. These conditions solubilise keratins. The insoluble residue was removed by centrifugation. Electron microscopy of the reconstituted fibrous proteins showed the characteristic $8 \mathrm{~nm}$ diameter filaments of keratin. Analysis of these proteins by SDSpolyacrylamide gel electrophoresis showed the same distribution of keratin polypeptides as obtained by Schlegel et al. ${ }^{6}$

The keratin proteins so obtained were used to produce an antibody. One milligram of total keratin protein was emulsified in Freund's complete adjuvant and injected intradermally into New Zealand white rabbits. The injection was repeated twice, at six week intervals, and the rabbits bled two weeks later. The antiserum was stored at $-20^{\circ} \mathrm{C}$.

\section{HISTOLOGICAL METHODS}

Paraffin-embedded tissues were obtained for each case. Using the PAP immunoperoxidase method ${ }^{7}$ a variety of normal human tissues was stained in addition to the test sections from maternal deaths. Endogenous peroxidase was blocked by incubation in freshly prepared $0.5 \%$ hydrogen peroxide in methanol for $30 \mathrm{~min}$. The antiserum was used at a dilution of $1 / 500$. The specificity of the immune serum was confirmed by preabsorption with keratin; $10 \mathrm{mg}$ of keratin absorbed completely $1 \mathrm{ml}$ of a 1/500 dilution of antiserum. Sections from all the maternal deaths were stained with haematoxylin and eosin and with Attwood's stain for amniotic debris. ${ }^{8}$ Sections of lung from 10 other unselected female necropsies were similarly examined.

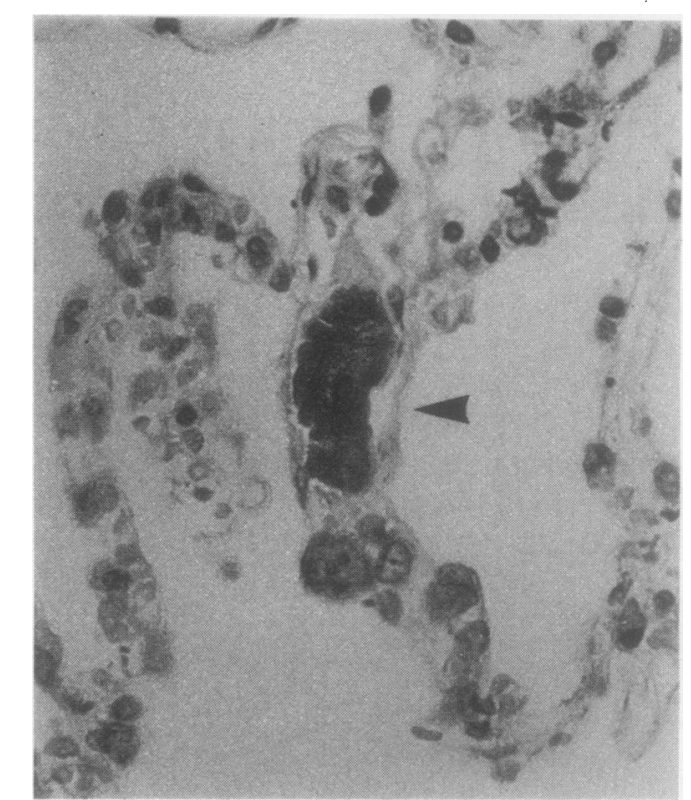

Section of lung showing squamous debris in vessel (arrowed). Immunoperoxidase $\times 475$.

\section{Results}

The antiserum stained positively sections from skin, $\stackrel{\mathbb{Q}}{\circ}$ vas deferens, bronchial epithelium and squamous $\overrightarrow{\overrightarrow{0}}$ tumours. These findings corroborate those of $\frac{3}{3}$ Schlegel et al. ${ }^{6}$ In the four maternal deaths, positive staining was seen easily within the pulmonary circulation. The amniotic squames stained a deep brown colour and could be identified by their shape and size. This made them easily distinguishable from nuclear staining and background staining. A positive control was present in the sections, as the basal cells of the bronchial epithelium stained positively though less intensely than amniotic squames (Figure).

Using Attwood's stain amniotic debris could easily be seen only in case 1 . No amniotic debris was $N$ identified in cases 2 and 4 and only a small amount $N$ in case 3. No squamous debris was detected in the 10 unrelated female necropsies. The only problem encountered was the presence of occasional contaminant squamous debris. This could be recognised as being outwith the plane of section.

\section{Discussion}

There are widely varying estimates of the incidence of amniotic fluid embolism. ${ }^{3}$ The DHSS in its report into maternal deaths $1973-75^{1}$ accepts a diagnosis of amniotic fluid embolism as a cause of death only 
when there are the clinical features of sudden intrapartum-or postpartum-collapse accompanied perhaps, by a fit, dyspnoea and cyanosis. Intrauterine death of the fetus is not uncommon. In addition there is usually DIC. These clinical features must be positively confirmed by the necropsy findings of amniotic fluid embolism within lung sections. Using these criteria they report an incidence of 7.8 deaths per million maternities.

With these clinical criteria, case 1 and perhaps case 4 would be diagnosed as amniotic fluid embolism. Using both the clinical and histological criteria, a diagnosis of amniotic fluid embolism would only have been accepted in case 1 . However, with the additional immunoperoxidase stain, amniotic fluid embolism can now be seen to be present and may be a contributory factor in all cases. This is an unexpected result from four consecutive maternal deaths occurring in one region. Two possible explanations may account for this high incidence. Either the clinical and initial necropsy diagnosis underestimated the contribution of amniotic fluid embolism or our more sensitive technique is detecting a trivial amount of embolism present even in normal deliveries.

In their study Roche and Morris 9 could detect amniotic debris only in a group of women who died of amniotic fluid embolism. The control group of women who died of eclamptic toxaemia, were not demonstrated as having amniotic debris within their pulmonary tree. In our study, case 3 , where the clinical and post-mortem diagnosis was of preeclampsia, squames were readily identified in the pulmonary circulation. This finding suggests that the incidence of amniotic fluid embolism has been underestimated. However, it has been shown that trophoblastic material does enter the circulation during every pregnancy, ${ }^{10}$ but so far this has not been recognised for amniotic fluid.

Another observation has been the coexistence in all our cases of DIC and amniotic fluid embolism. The diagnosis of DIC was originally attributed to pre-eclamptic toxaemia and/or infection in the latter three cases and to amniotic fluid embolism in case 1 .
It is possible that the amniotic fluid embolism played a more causal role in the formation of DIC in the latter three cases than previously appreciated but, on our data, no final conclusion can be drawn.

It is now possible to determine the true incidence of amniotic fluid emboli by this more sensitive method. By measuring the number of squames found, and relating that to the other clinical and histological data, it should also be possible to determine the importance and frequency of these emboli in causing maternal deaths.

We wish to thank Mr G King for his technical assistance. This study was supported by research funds of Aberdeen University.

\section{References}

' DHSS. Report on confidential enquiries into maternal deaths in England and Wales 1973-75. HMSO: 1979;14:93-7.

${ }^{2}$ Anonymous. Lancet 1979;ii:398-400.

${ }^{3}$ Attwood HD. Amniotic fluid embolism. In: Sommers SC, ed. Pathology annual 1972;7:145-72.

${ }^{4}$ Guidotti RJ, Grimes DA, Cates W. Fatal amniotic fluid embolism during legally induced abortion. Am J Obstet Gynecol 1981;141:257-61.

${ }^{5}$ Fuchs $\mathrm{E}$, Green $\mathrm{H}$. The expression of keratin genes in epidermis and cultured epidermal cells. Cell 1978;15:887-97.

- Schlegel R, Banks-Schlegel S, Pinkus G. Immunohistochemical localisation of keratin in normal human tissues. Lab Invest 1980;42:91-6.

' Sternberger LA, Hardy RP Jr, Cuculis SS, Meger HG. The unlabelled antibody enzyme method of immunohistochemistry. Preparation properties of soluble antigen-antibody complex (horse radish peroxidase--antihorse radish peroxidase) and its use in identification of spirochaetes. $J$ Histochem Cytochem 1976;18:325.

${ }^{8}$ Attwood HD. The histological diagnosis of amniotic fluid embolism. J Pathol Bacteriol 1958;76:211-5.

9 Roche WD, Morris HJ. Detection and significance of maternal pulmonary amniotic fluid embolism. Obstet Gynecol 1974;43:729-31.

${ }^{10}$ Douglas GW, Thomas L, Carr M, Cullen NM, Morris R. Trophoblast in the circulating blood during pregnancy. $A m J$ Obstet Gynecol 1959;78:960-73.

Requests for reprints to : Dr IWC Garland, Department of Pathology, University Medical Buildings, Foresterhill, Aberdeen AB9 2ZD, Scotland. 